\title{
Effect of antibacterial/adhesive approaches on bonding durability of fiber posts cemented with self-etch resin cement
}

\author{
Fereshteh Shafiei $^{1}$, Mahtab Memarpour ${ }^{1}$, Narges Vafamand ${ }^{2}$, Mahsa Mohammadi ${ }^{2}$
}

${ }^{1}$ DMD, MScD, Professor, Oral and Dental Disease Research Center, School of Dentistry, Shiraz University of Medical Sciences, Shiraz, Iran

${ }^{2}$ DDS, Dentist, Department of Operative Dentistry, School of Dentistry, Shiraz University of Medical Sciences, Shiraz, Iran

Correspondence:

Prevention of Oral and Dental Disease Research Center

School of Dentistry Shiraz University of Medical Sciences

Shiraz, Iran

memarpour@sums.ac.ir

\begin{abstract}
Shafiei F, Memarpour M, Vafamand N, Mohammadi M. Effect of antibacterial/adhesive approaches on bonding durability of fiber posts cemented with self-etch resin cement. J Clin Exp Dent. 2017;9(9):e1096102.

http://www.medicinaoral.com/odo/volumenes/v9i9/jcedv9i9p1096.pdf
\end{abstract}

Received: 28/04/2017

Accepted: 09/08/2017

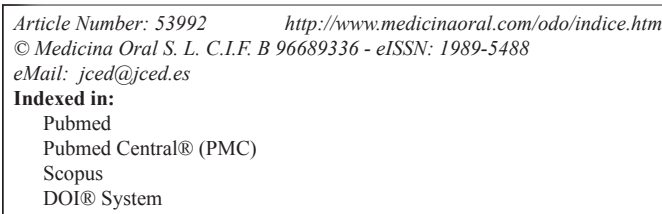

\begin{abstract}
Background: longevity of post-retained restoration is highly depended on bonding stability of fiber post (FP) to root dentin. This study evaluated the effect of different antibacterial/adhesive approaches on bonding durability of FPs luted into root canal with a self-etch cement.

Material and Methods: Seventy-two human maxillary central incisor roots were divided into six groups after endodontic treatment, based on the antibacterial/adhesive treatments as follows: 1)ED primer II (ED, control); 2) Clearfil Protect Bond (PB); 3) 2\% chlorhexidine (CH) pretreatment + ED primer II (CH+ED); 4) CH-incorporated into ED primer II (CH in ED); 5) CH pretreatment + Clearfil SE Bond (CH+SE); and 6)CH-incorporated into SE primer ( $\mathrm{CH}$ in SE). The FPs were then cemented using PanaviaF2.0. After micro-slicing the bonded root dentin, a push-out bond strength (PBS) test was performed immediately or after two years of water storage. Data were analyzed using ANOVA and post hoc Tukey tests $(\alpha=0.05)$.

Results: The effects of antibacterial/adhesive approach, time and interaction between the main factors were significant ( $p=0.01)$. There was no significant difference between the immediate groups, except between the CH+ED group (the lowest PBS) and PB and $\mathrm{CH}$ in SE groups (the highest PBS) $(p \leq 0.03)$. After aging, the same difference was observed $(p \leq 0.02)$; the control group exhibited a significantly lower PBS compared to the other groups $(p \leq 0.01)$, except for $\mathrm{CH}+\mathrm{ED}$. Aging significantly decreased PBS of all the groups $(p \leq 0.01)$; the control group exhibited the highest reduction.

Conclusions: $\mathrm{CH}$ incorporated into self-etch primers or in pretreatment step prior to two-step self-etch adhesive and antibacterial adhesive could improve bond stability of self-etch cemented fiber post. However, none of these was capable of inhibiting bond degradation over time.
\end{abstract}

Key words: Push-out bond strength, Fiber post, Chlorhexidine. 


\section{Introduction}

Numerous favorable properties of fiber posts (FP) have resulted in their widespread use for the restoration of endodontically treated teeth. In adhesive cementation of FPs establishment of a highly durable bond between resin cement and root dentin is an essential factor to provide a coronal seal and adequate retention (1).

Self-etch adhesive (SE) resin cements might be preferred to etch-and-rinse ones by clinicians due to simplification and less technique sensitivity (2). These systems do not need acid etching and rinsing. As a result, a thick and heavy secondary smear layer formed during post space preparation could remain, which possibly contains microorganisms. These, in addition to penetration of oral bacteria through coronal leakage, might jeopardize prognosis of root canal therapy (3). Therefore, use of antibacterial agents is a critical and important step during post luting procedures. Chlorhexidine $(\mathrm{CH})$ with excellent substantive antibacterial properties acts as a matrix-metalloproteinase (MMP) and cathepsin inhibitor, preserving collagen matrix of dentin $(4,5)$. Some studies reported bonding longevity of adhesive-cemented FPs following $\mathrm{CH}$ irrigation of post space in root canals with different results (6-8). However, this approach introduces an additional step to complex fiber post adhesive cementation, increasing chair-time. Although this paves the way for benefiting from better efficacy of antibacterial activity of $\mathrm{CH}$ in $2 \%$ concentration than lower concentrations (9) in the root canal, it might limit the efficacy of MMP inhibitory activity of $\mathrm{CH}$ due to simultaneous demineralization and resin penetration of SE cements into the smear layer-covered dentin.

On this basis, incorporating $\mathrm{CH}$ into acidic primers could be another approach to provision of positive effects of $\mathrm{CH}$ during adhesive cementation. Firstly, Hiraishi et al. demonstrated that incorporation of $1 \% \mathrm{CH}$ into $\mathrm{ED}$ Primer 2.0 exhibited significant antibacterial activity without any adverse effect on bond strength of Panavia F2.0 to dentin (10). This procedure could dissolve/infiltrate the smear layer along with $\mathrm{CH}$ penetration, consequently allowing facilitated interaction of $\mathrm{CH}$ with the activated MMPs by acidic monomers and inhibitory effect of $\mathrm{CH}$. This approach was found to have positive results in bonding durability of FPs bonded with Panavia F2.0 (11).

Quaternary ammonium compounds (QAC), as a main antibacterial agent incorporated into the adhesive systems, have a stable and durable antibacterial activity (12). MDPB is the combined QAC, and methacrylate group as an antibacterial monomer has a disinfecting ability in an uncured state. After copolymerization with other monomers, it inhibits bacterial growth on its surface $(12,13)$. The more antibacterial effectiveness of experimental solution containing MDPB than $\mathrm{CH}$ solution on bacteria related to endodontic infections has been clarified by a recent study (14). This monomer has been incorporated into a commercially available two-step SE, Clearfil Protect Bond by Kuraray (12). This adhesive exhibited a stable bond strength to coronal dentin after one year and better antibacterial activity compared to the other antibacterial agents incorporated $\mathrm{CH}$ and glutaraldehyde) (15). Recently MDPB has been found to inhibit MMPs and cathepsins (16).

To date, no study has compared bonding longevity of SE cement to intraradicular dentin following different antibacterial applications during fiber post cementation.

The aim of this study was to test the null hypothesis that various adhesive/antibacterial approaches have no effects on bonding performance of an SE cement in root canal space immediately and at long term.

\section{Material and Methods}

Seventy-two sound human maxillary central incisors with approximately similar size and anatomic shape were selected. The roots of the selected teeth were free of cracks and root resorption and were mature. They were stored in $0.5 \%$ chloramine- $\mathrm{T}$ solution at $4{ }^{\circ} \mathrm{C}$ and then in distilled water. They were used following informed consent from the patients and approval of the study protocol by the local Ethics Committee. The roots were cut to obtain a uniform length of $15 \mathrm{~mm}$ from the apex and then endodontically treated.

After one week of storage in water, post spaces were prepared to create a standardized depth of $10 \mathrm{~mm}$ using the respective drills provided by the post manufacturer. These procedures were verified by radiographs.

FPs (Glassix Post, H.Nordin, SA, Chailly-Montreux, Switzerland) were tried in the prepared canals for a passive fit. The post surfaces were cleaned with ethanol, air-dried and then silanized. The specimens were then randomly divided into six groups $(\mathrm{n}=12)$ based on the antibacterial/adhesive treatments of the root dentin as follows:

Group 1 (ED): ED primer II was applied as a control.

Group 2 (PB): A two-step SE antibacterial adhesive, Clearfil Protect Bond, was applied.

Group $3(\mathrm{CH}+\mathrm{ED})$ : 2\% chlorhexidine $(\mathrm{CH})$ solution (Consepsis, Ultradent, USA) was applied using an endodontic brush for $60 \mathrm{~s}$. Then the canals were dried with paper points and ED primer II was applied.

Group 4 (CH in ED): Chlorhexidine diacetate (SigmaAldrich, St. Louis, MO ,USA) was directly added to a mixture of ED primer II A and B to prepare the primer containing $1 \mathrm{wt} \%$.

Group $5(\mathrm{CH}+\mathrm{SE})$ : $\mathrm{CH}$ solution was applied similar to that in group 3. Then, a two-step SE (Clearfil SE Bond) was applied.

Group 6 (CH in SE): The CH-incorporated Clearfil SE primer was prepared similar to that in ED primer and then Clearfil SE Bond was applied. 
During adhesive post cementation, the roots were held in a moist gauze sponge to maintain their moisture content. All the bonding steps were carried out by the same operator, according to manufacturer's instructions (Table 1). In all the groups, the mixed Panavia F2.0 was through the following formula $\pi(\mathrm{R}+\mathrm{r})[(\mathrm{h} 2+(\mathrm{R}-\mathrm{r}) 2], 0.5$ where $\mathrm{R}$ and $\mathrm{r}$ represent the coronal and the apical root canal radii, respectively, and $h$ is the thickness of the slice.

All the debonded root slices were assessed under a ste-

Table 1: Materials and application procedures used in the current study.

\begin{tabular}{|c|c|c|}
\hline $\begin{array}{l}\text { Adhesive - cement system/ } \\
\text { Manufacturer/Lot\# }\end{array}$ & Application mode & Composition \\
\hline $\begin{array}{l}\text { ED primer II - Panavia F2.0/ } \\
\text { Kuraray, Osaka Japan } \\
\text { A: 00198A, B: 00324F-A: } \\
\text { 00588A,B:00116C }\end{array}$ & $\begin{array}{l}\text { Mixing ED Primer II (A and B),applying } \\
\text { for } 30 \mathrm{~s} \text {, drying with paper points and air } \\
\text { stream, cementing with mixed Panavia } \\
\text { F2.0. }\end{array}$ & $\begin{array}{l}\text { Primer A: HEMA, 10-MDP, N- } \\
\text { methacryloxyl-5-aminosalicyclic } \\
\text { acid, water, accelerator } \\
\text { Primer B: N-methacryloxyl-5- } \\
\text { aminosalicyclic acid, accelerator, } \\
\text { water, sodium } \\
\text { benzene sulphinate }\end{array}$ \\
\hline $\begin{array}{l}\text { Clearfil Protect Bond/Kuraray, } \\
\text { Osaka, Japan } \\
\text { Primer:01039A, Bond:01550A }\end{array}$ & $\begin{array}{l}\text { Applying primer for } 20 \text { s,drying with } \\
\text { paper point and air stream,apply Bond } \\
\text { to the root canal and then create uniform } \\
\text { film using air stream,light polymerize for } \\
\qquad 40 \mathrm{~s} \text {. }\end{array}$ & $\begin{array}{c}\text { Primer: HEMA, hydrophilic } \\
\text { Dimethacrylate, MDP, water, } \\
\text { 12-methacryloyloxy dodecyl } \\
\text { pyridinium bromide } \\
\text { Bond: MDP, Bis-\%MA, HEMA, } \\
\text { Hydrophobic Dimethacrylate, } \\
\text { comphorquinone, N,N-diethanol-P- } \\
\text { Toluidine Fluoride }\end{array}$ \\
\hline $\begin{array}{l}\text { Clearfil SE Bond/ Kuraray, } \\
\text { Osaka, japan } \\
\text { Primer: 01226A, Bond: 01851A }\end{array}$ & $\begin{array}{l}\text { Applying primer for } 20 \mathrm{~s} \text {,drying with } \\
\text { paper point and air stream,apply Bond } \\
\text { to the root canal and then create uniform } \\
\text { film using air stream,light polymerize for } \\
\qquad 40 \mathrm{~s} \text {. }\end{array}$ & $\begin{array}{c}\text { Primer: water, MDP, HEMA, } \\
\text { camphorquinone, hydrophilic } \\
\text { dimethacrylate } \\
\text { Bond: MDP, Bis-GMA, HEMA, } \\
\text { Comphorquinone hydrophobic } \\
\text { dimethacrylate N, N. } \\
\text { diethanol-toluidine }\end{array}$ \\
\hline
\end{tabular}

applied on the post surface and to the post space. The post was immediately seated with a slight vibratory motion and held under finger pressure; after removal of the excess cement, light-curing was performed for $60 \mathrm{~s}$ at $600 \mathrm{~mW} / \mathrm{cm}^{2}$ using a light-curing unit (VIP Junior, Bisco, Schaumburg, IL, USA). After one week of water storage, each root was sectioned to obtain seven 1-mmthick slices by using a slow-speed cutting machine (Mecatome T201 A, Presi, Grenoble, France). The first coronal slice was excluded. In half of the roots from each group ( $\mathrm{n}=6,36$ slices), the push-out test was performed immediately. The other half of each group was stored in distilled water containing $0.4 \%$ sodium azide for two years before assessing the long-term bond strength.

The slices were submitted to a compressive load in a universal testing machine (Zwick, Roell, Ulm, Germany) at $0.5 \mathrm{~mm} / \mathrm{min}$ on the center or the apical surface of the post in an apico-coronal direction until the shear stress applied along the bonded interface dislodged the post. The load of debonding in Newton (N) was divided by the adhesive interface area and push-out bond strength was recorded in MPa. The bonded area was calculated reomicroscope (Carl Zeiss Inc, Oberkochen, Germany) at $\times 40$ and classified as follows: 1) adhesive failure in the dentin; 2) cohesive failure in the cement; 3 ) adhesive failure between the dentin and cement; 4) adhesive failure between the post and cement; and 5) mixed failures consisting of a combination of two or more failure modes.

The representative specimens of failure modes were prepared for scanning electron microscopy (SEM; EM3200, KYKY, Beijing, China) observations of the failure patterns as shown in Figure 1.

\section{Results}

Table 2 presents the means and standard deviations (in $\mathrm{MPa}$ ) of push-out bond strengths (PBS) of the six groups. Two-way ANOVA showed that the effects of adhesive/ antibacterial agent, time and interaction between the two main factors were significant $(p \leq 0.01)$.

For each time interval, one-way ANOVA and post hoc Tukey tests were used to compare the PBS of the six groups. Student's t-test was used to compare the effect of aging on PBS in each group $(\alpha=0.05)$. 


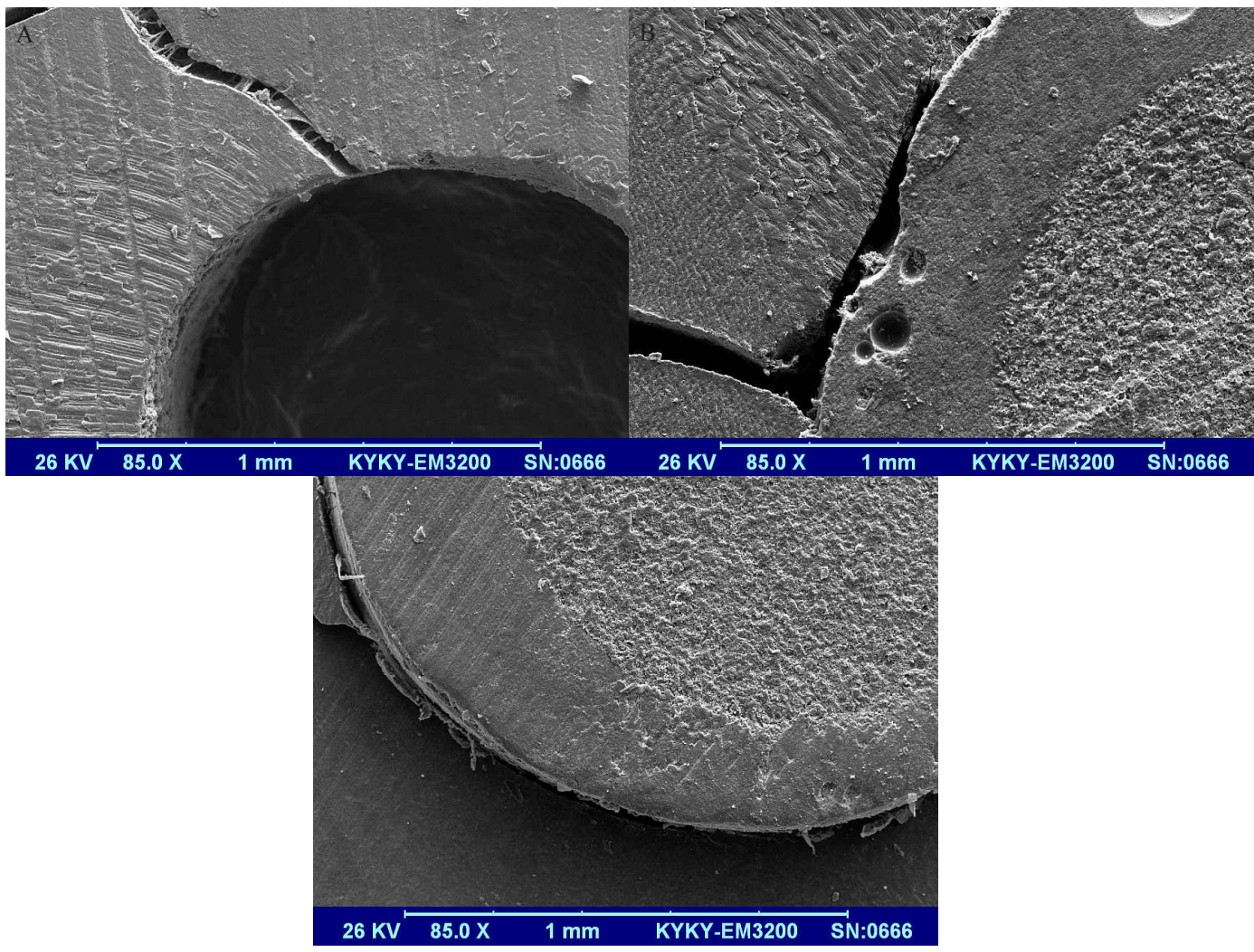

Fig. 1: Scanning electron micrograph of representative failure modes: A and B) Mixed failures including adhesive failure between the resin cement and root dentin along with cohesive failure in the root dentin, (A): showing no remaining resin on root dentin wall and (B): showing a gap-free interface between the resin cement and post. C) Adhesive failure between the resin cement and the root dentin.

Table 2: Push-out bond strength (mean $\pm \mathrm{SD}$ ) of Panavia F2.0 in the six groups at two time intervals and failure mode ( $\mathrm{n}=36$ ).

\begin{tabular}{|c|lc|lc|}
\hline \multirow{2}{*}{ Groups } & \multicolumn{2}{|c|}{ Immediate } & \multicolumn{2}{c|}{12 months } \\
\cline { 2 - 5 } & Mean $\pm \mathrm{SD}(\mathrm{MPa})$ & failure $\mathrm{Cd} / \mathrm{Cc} / \mathrm{Ac}-\mathrm{d} / \mathrm{Ac}-\mathrm{p} / \mathrm{M}$ & Mean $\pm \mathrm{SD}(\mathrm{MPa})$ & failure Cd/Cc/Ac-d/Ac-p/M \\
\hline 1 & $14.25 \pm 2.24^{\mathrm{aB}}$ & $3 / 2 / 4 / 1 / 26$ & $8.53 \pm 2.20^{\mathrm{bA}}$ & $1 / 2 / 20 / 2 / 11$ \\
\hline 2 & $16.29 \pm 3.77^{\mathrm{aB}}$ & $2 / 1 / 5 / 4 / 24$ & $10.46 \pm 3.72^{\mathrm{bA}}$ & $1 / 0 / 13 / 2 / 20$ \\
\hline 3 & $13.09 \pm 2.14^{\mathrm{aA}}$ & $3 / 0 / 6 / 6 / 2$ & $11.91 \pm 3.04^{\mathrm{bB}}$ & $0 / 1 / 7 / 4 / 24$ \\
\hline 4 & $13.87 \pm 1.84^{\mathrm{aAB}}$ & $1 / 2 / 5 / 5 / 23$ & $11.00 \pm 2.14^{\mathrm{bB}}$ & $2 / 1 / 9 / 5 / 19$ \\
\hline 5 & $15.52 \pm 3.66^{\mathrm{aAB}}$ & $0 / 1 / 7 / 4 / 24$ & $13.47 \pm 1.97^{\mathrm{bB}}$ & $2 / 1 / 8 / 4 / 21$ \\
\hline 6 & $15.92 \pm 3.60^{\mathrm{aB}}$ & $2 / 3 / 4 / 8 / 19$ & & \\
\hline
\end{tabular}

Groups: 1: ED primer 11 (control) 2: Protect bond 3: $\mathrm{CH}+\mathrm{ED}$ 4: $\mathrm{CH}$ in ED 5: $\mathrm{CH}+\mathrm{SE}$ 6: $\mathrm{CH}$ in SE. Failure: Cd: cohesive in dentin; Cc: cohesive in cement, Ac-d: adhesive between the cement and the dentin; Ac-p: adhesive between the cement and the post; M: mixed failures. Within each line (group), different lower case letters indicate statistically significant difference; within each column (time interval) different capital letters indicate statistically significant difference. 
CH+ED (13 \pm 2.1$)$ exhibited a lower immediate PBS compared to those of $\mathrm{PB}(16.29 \pm 3.7)$ and $\mathrm{CH}$ in the SE $(15.92 \pm 3.6)$ groups ( $p=0.01$ and $p=0.03$, respectively). There was no significant difference between the other groups $(p>0.05)$.

After aging, PBS of the ED control group (8.53 \pm 2.2$)$ was significantly lower than those of the other groups $(p \leq 0.01)$, except for the $\mathrm{CH}+\mathrm{ED}$ group (10.46 \pm 3.7$)$. Similar to the immediate PBS group, the latter group yielded a lower PBS than those of the PB group (12.8 \pm 2.2$)$ and $\mathrm{CH}$ in the SE group with the highest PBS $(13.47 \pm 1.9)$ ( $p=0.02$ and $\mathrm{p}=0.002$, respectively). PBS of the latter group was significantly higher than the $\mathrm{PBS}$ of $\mathrm{CH}+\mathrm{SE}$ group $(11 \pm 2.1)(p=0.01)$.

Aging significantly decreased PBS of all the groups with different extents $(p \leq 0.01)$. The highest absolute reduction was recorded for the control (ED) group (11.31), followed by the $\mathrm{CH}+\mathrm{SE}$ (4.95) and PB (3.64) groups. The other three groups exhibited a comparable absolute reduction (2.8).

The results of failure analysis of the six study groups revealed that the majority of failure modes were mixed failures in immediate groups. After aging the modes of failure were mostly adhesive failures between the root dentin and cement in the ED primer (control) group, while in the other groups mixed failure was the predominant failure mode (Table 2).

\section{Discussion}

According to the results of this study, there was a significant difference between immediate PBS of different adhesive/antibacterial procedures, rejecting part of the tested null hypothesis. Although incorporation of $\mathrm{CH}$ into ED primer/SE primer (1\%) or its separate application $(2 \%)$ had no significant effect on initial bonding ability of Panavia F2.0, separate application of $\mathrm{CH}$ before ED primer led to significantly lower PBS compared to those of only PB and $\mathrm{CH}$ in the SE groups. A similar significant difference was observed after aging. These two groups and $\mathrm{CH}+\mathrm{SE}$ with immediate comparable $\mathrm{PBS}$ (16.29-15.52) are two-step SE systems that have a separately applied resin layer with higher hydrophobicity. It was reported that the additional light-cured resin layer applied on ED primer might provide additional free radicals to improve the rate and extent of polymerization of ED primer and contribute to reduced permeability of the adhesive layer (17). ED primer is a single-step SE adhesive that exhibits some permeability due to its high content of hydrophilic and acidic monomer components and the lack of the subsequent application of a hydrophobic resin layer (17). In vivo permeability of these simplified adhesives was previously documented even in root-treated dentin. This might negatively influence bonding of dual-cured resin cement to the root dentin. This permeability could also occur in vitro even without a perfusion system because the teeth were retrieved from the water storage medium and bonded in their normal hydrated state (18). It was suggested that ED primer is essential for adequate polymerization of Panavia F; even in the absence of light-curing, not for bonding to dentin.

Another explanation for improved bond strength of Panavia F2.0 to coronal dentin after adding the resin layer reported by Carvarlho et al. was the relief of shrinkage stress induced by the resin cement (17). The positive effect of this factor could be more relevant in deep, narrow and confined root canal space with the extremely high C-factor.

Our results revealed that direct incorporation of $1 \% \mathrm{CH}$ into ED primer had no adverse effect on immediate PBS. A similar result was reported by Zhou et al. during bonding of FP with Panavia F2.0 to root dentin (11). Zhou et al. reported no adverse effects of adding $\mathrm{CH}$ at $\leq 1 \mathrm{wt} \%$ into self-etch primer of Clearfil SE Bond on the immediate bond strength. These authors demonstrated that this combination could preserve the bond strength to coronal dentin after 12 months of water aging (19). This positive effect was related to the MMP inhibitory effect of incorporated of CH into SE primer (20). No study has evaluated this approach in root canals.

Based on the results of the current study, after aging the coronal group (ED primer) exhibited a significantly lower PBS compared to those of the other groups with antibacterial agent except for the $\mathrm{CH}+\mathrm{ED}$ group. In this context, the diminished PBS observed in all the groups was the highest for ED primer group. Therefore, the other part of the tested hypothesis could not be confirmed.

Two main factors are involved in adhesive bond degradation: collagen degradation and hydrolysis of hydrophilic ionic resin monomers in the simplified primer/adhesive $(21,22)$. The results of this study might be attributed to the factors mentioned above during the accelerated aging via direct water exposure of micro-sliced specimens. A rapid degradation process might have occurred following rapid water diffusion through the small surface area of the adhesive interface (22). In particular, difficulties in bonding and penetration of curing light into root canal compared with coronal dentin could create a weak polymer with low degree of polymerization (11), enhancing resin degradation. The highest PBS was obtained in $\mathrm{CH}$ in the SE group, which was significantly higher than that in the $\mathrm{CH}+\mathrm{SE}$ group. This might be attributed to the partial protective effect of $\mathrm{CH}$ on bonding longevity through an inhibitory effect on MMPs. This effect appeared to depend on application mode of $\mathrm{CH}$ with each adhesive so that $\mathrm{CH}$ incorporated into Clearfil SE primer was more effective than separately applying $\mathrm{CH}$ before SE primer. The curing of a hydrophobic resin layer on the hybrid layer treated with $\mathrm{CH}$ can cover and 
preserve $\mathrm{CH}$ at the adhesive interface to prolong its beneficial effects (23).

Hiraishi et al. demonstrated that $\mathrm{CH}$ pretreatment might be an adverse effect on immediate bonding performance of Panavia F2.0 cement (24). These authors believed that this might be attributed to the moisture control in the coronal dentin treated with $\mathrm{CH}$ rather than to the intrinsic properties of $\mathrm{CH}$ (24). The incorporation of $\mathrm{CH}$ into primer can simplify bonding procedures along with lower technique sensitivity. This aspect might have partly contributed to our results in relation to the ineffectiveness of $\mathrm{CH}$ application prior to ED primer in diminishing PBS loss over time. Lindblad et al. found no effect of $\mathrm{CH}$ pretreatment on immediate and one-year PBS of FPs with ER and self-adhesive resin cements in root canal space, but $\mathrm{CH}$ changed the fracture pattern from pure adhesiveto-dentin failure to mixed and cohesive-in-dentin failures $(25,26)$. In the current study, despite similar failure modes in the immediate groups, distribution of failure modes was different among the groups after aging. In this context, in the control group the adhesive failure between the cement and root dentin was predominant, while mixed failure was dominant for the other groups, possibly indicating the positive effect of $\mathrm{CH}$ and the antibacterial effect of adhesive on bonding longevity. Conflicting results have been reported regarding MMPinhibitory activity of MDPB and bond stability of the antibacterial adhesive (27). Consistent with our results, some studies have shown no adverse effects of $2 \% \mathrm{CH}$ pretreatment on immediate PBS of posts bonded with SE adhesive cements $(6,28)$. However, an adverse effect of $\mathrm{CH}$ on CSE, especially in the apical third of the root, was reported (2). The beneficial effect of $\mathrm{CH}$ on preserving PBS of fiber post bonded with SE adhesive (CSE) was confirmed in two one-year studies $(7,8)$. Contrary to our results, in these studies PBS of $\mathrm{CH}$-pretreated groups did not significantly decrease after one year; the full length of bonded roots were water-aged and then submitted to slice preparation for PBS testing. However, the bonded roots of the current study were first sectioned and then water-aged. The different aging processes used might explain the differences in the results.

Although long-term water storage of root micro-slices and the subsequent push-out test cannot closely mimic clinical aging, this experimental set-up was previously designed to evaluate the role of anti-MMP property of $\mathrm{CH}$ and the other agent on bonding stability of FPs to radicular dentin $(11,29)$. Furthermore, similar patterns of hybrid layer degradation have been found in vivo from the base of adhesive-bonded cavities (30). A study by Zhou et al. on $\mathrm{CH}$ showed the positive effect of $1 \% \mathrm{CH}$ incorporated into ED primer on bonding durability of FPs for 18 months of water storage. However, a significant decrease in PBS was observed in the $1 \% \mathrm{CH}$ group (11). This finding was supported by the results of the current study.

The long-term effect of $\mathrm{CH}$ might be attributed to its high substantivity and its electrostatic binding to the mineralized and demineralized dentin (30). Nevertheless, $\mathrm{CH}$ might be released from the adhesive interface over time, decreasing its anti-MMP effect (20). This may be higher in this experimental set-up than in vivo condition due to a higher direct contact of sectioned adhesive interface with water during water storage. The antibacterial adhesive does not release the antibacterial component over time. This study was conducted using single SE resin cement. The adhesives used instead of ED primer II were from the same manufacturer as Panavia F2.0, preventing any incompatibility between different products. The results of the present study cannot be generalized to other adhesive resin cements with different chemistry.

\section{Conclusions}

Considering the limitations of this in vitro study, it can be concluded that $\mathrm{CH}$ incorporated into self-etch primers or in pretreatment step prior to application of Clearfil SE Bond and antibacterial adhesive could improve bond stability of FPs in root canal space. Nevertheless, none of these antibacterial protocols was capable of completely inhibiting bond degradation in the long term.

\section{References}

1. Bitter K, Kielbassa AM. Post-endodontic restorations with adhesively luted fiber-reinforced composite post systems: a review. Am J Dent. 2007;20:353.

2. Wang L, Pinto T, Silva L,Araújo DF, Martins LM, Hannas AR, et al. Effect of $2 \%$ chlorhexidine digluconate on bond strength of a glassfibre post to root dentine. Int Endod J. 2013;46:847-854.

3. Hayashi M, Takahashi Y, Hirai M, Iwami Y, Imazato S, Ebisu S. Effect of endodontic irrigation on bonding of resin cement to radicular dentin. Eur J Oral Sci. 2005;113:70-76.

4. Basrani B, Santos JM, Tjäderhane L, Grad H, Gorduysus O, Huang $\mathrm{J}$, et al. Substantive antimicrobial activity in chlorhexidine-treated human root dentin. Oral Surg Oral Med Oral Pathol Oral Radiol Endod. 2002;94:240-245.

5. Scaffa PM, Vidal CM, Barros N, Gesteira TF, Carmona AK, Breschi L, et al. Chlorhexidine inhibits the activity of dental cysteine cathepsins. J Den Res. 2012;91:420-425.

6. Bitter K, Hambarayan A, Neumann K, Blunck U, Sterzenbach G. Various irrigation protocols for final rinse to improve bond strengths of fiber posts inside the root canal. Eur J Oral Sci. 2013;121:349-354.

7. Cecchin D, de Almeida JFA, Gomes BP, Zaia AA, Ferraz CCR. Effect of chlorhexidine and ethanol on the durability of the adhesion of the fiber post relined with resin composite to the root canal. J Endod. 2011;37:678-683.

8. Cecchin D, Farina AP, Giacomin M, Vidal CdMP, Carlini-Júnior B, Ferraz CCR. Influence of chlorhexidine application time on the bond strength between fiber posts and dentin. J Endod. 2014;40:2045-2048. 9. Mohammadi Z, Abbott P. The properties and applications of chlorhexidine in endodontics. Int Endod J. 2009;42:288-302.

10. Hiraishi N, Yiu C, King N, Tay F. Effect of chlorhexidine incorporation into a self-etching primer on dentine bond strength of a luting cement. J Dent. 2010;38:496-502.

11. Zhou J, Yang X, Chen L, Liu X, Ma L, Tan J. Pre-treatment of radicular dentin by self-etch primer containing chlorhexidine can improve fiber post bond durability. Dent Mater J. 2013;32:248-255. 
12. Cocco AR, da Rosa WLdO, da Silva AF, Lund RG, Piva E. A systematic review about antibacterial monomers used in dental adhesive systems: Current status and further prospects. Dent Mater. 2015;31:1345-1362.

13. Izutani N, Imazato S, Nakajo K, Takahashi N, Takahashi Y, Ebisu $\mathrm{S}$, Russell RR, et al. Effects of the antibacterial monomer 12-methacryloyloxydodecylpyridinium bromide (MDPB) on bacterial viability and metabolism. Eur J Oral Sci. 2011;119:175-181.

14. Hirose N, Kitagawa R, Kitagawa H, Maezono H, Mine A, Hayashi $\mathrm{M}$, et al. Development of a Cavity Disinfectant Containing Antibacterial Monomer MDPB. J Dent Res. 2016;95:1487-1493.

15. André CB, Gomes BPFA, Duque TM, Stipp RN, Chan DC, Ambrosano GM, et al. Dentine bond strength and antimicrobial activity evaluation of adhesive systems. J Dent. 2015;43:466-475.

16. Tezvergil-Mutluay A, Agee KA, Mazzoni A,Carvalho RM, Carrilho M, Tersariol IL, et al. Can quaternary ammonium methacrylates inhibit matrix MMPs and cathepsins? Dent Mater. 2015;31:e25-e32.

17. Carvalho R, Pegoraro T, Tay F, Pegoraro L, Silva N, Pashley DH. Adhesive permeability affects coupling of resin cements that utilise self-etching primers to dentine. J Dent. 2004;32:55-65.

18. Chersoni S, Acquaviva GL, Prati C, Ferrari M, Grandini S, Pashley $\mathrm{DH}$, et al. In vivo fluid movement through dentin adhesives in endodontically treated teeth. J Dent Res. 2005;84:223-227.

19. Zhou J, Tan J, Yang X, Cheng C, Wang X, Chen L. Effect of chlorhexidine application in a self-etching adhesive on the immediate resindentin bond strength. J Adhes Dent. 2010;12:27.

20. Zhou J, Tan J, Chen L, Li D, Tan Y. The incorporation of chlorhexidine in a two-step self-etching adhesive preserves dentin bond in vitro. J Dent. 2009;37:807-812.

21. Reis A, Carrilho M, Breschi L, Loguercio A. Overview of clinical alternatives to minimize the degradation of the resin-dentin bonds. Oper Dent. 2013;38:E103-E127.

22. Liu Y, Tjäderhane L, Breschi L,Mazzoni A, Li N, Mao J, et al. Limitations in bonding to dentin and experimental strategies to prevent bond degradation. J Dent Res. 2011;90:953-968.

23. Hashimoto M, Ohno H, Sano H, Tay FR, Kaga M, Kudou Y, et al. Micromorphological changes in resin-dentin bonds after 1 year of water storage. J Biomed Mater Res A. 2002;63:306-311.

24. Hiraishi N, Yiu C, King N, Tay F. Effect of $2 \%$ chlorhexidine on dentin microtensile bond strengths and nanoleakage of luting cements. J Dent. 2009;37:440-448.

25. Lindblad RM, Lassila LV, Salo V, Vallittu PK, Tjäderhane L. Effect of chlorhexidine on initial adhesion of fiber-reinforced post to root canal. J Dent. 2010;38:796-801.

26. Lindblad RM, Lassila LV, Salo V, Vallittu PK, Tjäderhane L. One year effect of chlorhexidine on bonding of fibre-reinforced composite root canal post to dentine. J Dent. 2012;40:718-722.

27. Tjäderhane L, Nascimento FD, Breschi L, Mazzoni A, Tersariol IL, Geraldeli S, et al. Strategies to prevent hydrolytic degradation of the hybrid layer-a review. Dent Mater. 2013;29:999-1011.

28. Martinho FC, Carvalho CAT, Oliveira LD, de Lacerda AJ, Xavier $\mathrm{AC}$, Augusto MG, et al. Comparison of different dentin pretreatment protocols on the bond strength of glass fiber post using self-etching adhesive. J Endod. 2015;41:83-87.

29. Shafiei F, Yousefipour B, Bassir M. Effect of Carbodiimide on bonding durability of adhesive-cemented fiber posts in root canals. Oper Dent. 2016;41:432-440.

30. Carrilho MR, Carvalho RM, Sousa EN, Nicolau J, Breschi L, Mazzoni A, et al. Substantivity of chlorhexidine to human dentin. Dent Mater. 2010;26:779-785.

\section{Acknowledgements}

The authors wish to thank the Vice-Chancellory of Research of Shiraz University of Medical Science, Shiraz, Iran, for supporting this research, grant number No\# 10995. The authors also thank Dr. M. Vossoughi of the Dental Research Development Center in Shiraz Dental School for the statistical analysis. This article is based on the thesis by Dr. Narges Vafamand and Mahsa Mohammadi.

\section{Conflicts of Interest}

The authors declare that no competing interests exist. 